\title{
Using Polarization features of visible light for automatic landmine detection
}

\author{
Wim de Jong and John Schavemaker \\ TNO Defence, Security and Safety \\ Wim.deJong@tno.nl; John.Schavemaker@tno.nl
}

\section{Introduction}

This chapter describes the usage of polarization features of visible light for automatic landmine detection. The first section gives an introduction to landmine detection and the usage of camera systems. In section 2 detection concepts and methods that use polarization features are described. Section 3 describes how these detection concepts have been tested and evaluated. The results of these tests are given in section 4 . Conclusions from these results are given in section 5 .

\section{Landmine detection with camera systems}

Landmines as left behind after military or civil conflicts are a huge problem. The main current practice to clear mine fields is by prodding and by use of a metal detector. Prodding is very time consuming, dangerous and demands a lot of concentration from the deminer. The metal detector is used to find all metal objects. However landmines that contain little or no metal also exist and moreover, in former battle zones a lot of metal shrapnel is left behind. The lack of sophisticated tools that are reliable makes the process of demining very slow. To improve the clearance speed in the future, many nations have put effort in landmine-detection research.

The research on sensor systems focuses on three main topics [1, 2]. Firstly, the development of new sensors. Secondly, the improvement of existing sensors. The third research topic is the integration of these sensors into a sensorfusion system. The use of one sensor is generally believed to be insufficient for landmine detection meeting the requirements of humanitarian demining (100\% detection) for the reason that a single sensor has a false-alarm rate which is too high or a detection rate which is too low. The goals of sensorfusion are to reduce the probability of false alarms $P($ fa $)$, to increase the probability of detection $P(\mathrm{~d})$, or to improve a combination of both.

\subsection{Polarization camera}

One of the sensors that is considered for a multi-sensor landmine detection system is the thermal infrared (TIR) camera [3]. TIR cameras are able to de-

Page: 1 job: version1.2 macro: svmult.cls date/time: 31-Aug-2005/9:50 
tect small temperature differences (as low as $15 \mathrm{mK}$ ). Landmines often have different heat conductivity and heat capacity compared to the soil and the vegetation around them. Due to these differences in thermal properties, differences in temperature between a landmine and the background may develop when the soil is heated or cooled down. However, TIR images of landmines in natural scenes contain clutter, since other (natural) objects like trunks, holes, and rocks also may have different thermal properties compared to the background. In the visual spectrum it is well known that unpolarized light reflected from a smooth surface becomes polarized [4]. This is also true for TIR radiation. However, for TIR radiation not only the reflection, but also the emission is polarized. Since in general the surfaces of landmines are smoother than the surfaces found in a natural background, the presence of significantly polarized TIR radiation is an extra indication for landmines (or other non-natural objects). Using a polarization setup, the performance of the TIR camera can be improved and thus have a larger contribution to the multi-sensor system $[5,6,7]$.

When compared to a TIR polarization camera a VIS polarization camera is in general much cheaper and more robust. The drawback that buried land mines can not be detected with a VIS polarization camera is in some applications less important. This chapter only deals with the automatic detection of landmines using a VIS polarization camera.

\subsection{The use of VIS polarization in area reduction}

Reduction of the suspected area (before close-in detection is started) is very important in mine-clearance operations, irrespective of the detection technique (prodding or using an advanced multi-sensor system). Area reduction is the process through which the initial area indicated as contaminated (during a general survey) is reduced to a smaller area. Area reduction consists mainly of collecting more reliable information on the extent of the hazardous area.

Mechanical methods to reduce the area for mine clearance are among others rollers and flails. So called Pearson rollers [8], mounted on an armored commercial front-end loader are sometimes used to rapidly reduce areas adjacent to locations where anti-personnel (AP) landmines are suspected. Since the AP mine rollers have proven to be successful within humanitarian mine clearance, The HALO Trust has developed an anti-tank (AT) mine roller unit to be mounted on a front-end loader like the AP mine roller. The AT roller system is designed to detonate anti-tank mines, but each detonation of an AT mine will incur a down time for repair of the rollers, since some damage is expected on an AT mine detonation.

In order to minimize the amount of physical AT mine detonations, a camera system is developed that uses polarization properties of visual light to reach a high-detection rate of AT mines, combined with a low false-alarm rate. The camera system is fitted to the roof of the vehicle and is forward

Page: 2 job: version1.2 macro: svmult.cls date/time: 31-Aug-2005/9:50 
looking. The goal is to detect as many surface-laid AT mines as possible and to stop the vehicle before the roller detonates the mine. Contrary to close-in mine detection, $100 \%$ detection is not a requirement in this scenario, since mines missed by the camera will be detonated by the rollers. For some applications a very low false-alarm rate (less than 3 alarms per $100 \mathrm{~m}$ forward movement of the vehicle) combined with a moderate detection rate would be a valuable system. The following list presents some possible camera system concepts and the assumptions they have on the camera image modality:

- Black and white (BW), visible light, video camera;

- color, visible light, video camera;

- BW, visible light, video camera with polarization;

- Color, visible light, video camera with polarization.

All mine-detection camera systems are based on assumptions regarding the appearance of landmines for image processing and landmine detection. The above-mentioned camera concepts of a visible-light camera with or without polarization have different mine-detection capabilities.

\section{Detection}

In addition to the conventional way of detecting mines using image intensity [9] and/or color features, an additional way is created by the use of polarizers. Polarization can add significantly to the systems robustness and its detection performance $[5,6,7]$, especially in the case of detecting artificial objects within a natural background. However, the performance of polarization features depends more on the operating conditions than the color or intensity features, which are more invariant to those conditions. For example, the position of the sun (both azimuth and elevation), viewing angle with respect to the sun position, and weather conditions (daylight, clouds) have an impact on the use of polarization. Extensive tests have given some insights into the application of polarization system and its effect on the system characteristics.

\subsection{Detection concepts}

The mine-detection concept that we propose should combine features and cues from different image-processing techniques:

Color and/or intensity analysis: Detection is based on object color [10] or intensity contrast with the surrounding background [9]. The contrast threshold is defined by global or local image statistics (mean and standard deviation) of the image.

Polarization analysis: Objects are detected based on their polarization contrast with the surrounding background [7].

Page: 3 job: version1.2 macro: svmult.cls date/time: 31-Aug-2005/9:50 
Edge detection and grouping: Straight or partly circular edges are extracted because they can indicate artificial objects. The subsequent step groups edges into hypothetical artificial objects (e.g. using an edge-based Hough transform [11]).

For this concept, each image-processing technique results in a set of possible object detections with computed color, edge or polarization features for which additional features (e.g., morphological) can be calculated. The resulting set of objects is put into a pattern-recognition classifier that performs the final mine detection based on all computed features and previously learned examples. In order to ensure robustness of this classifier it is important that the image-processing techniques deliver invariant features. For example, scale invariance [12] can be found with proper camera calibration.

\subsection{Detection methods}

For the tests that are described in section 3 we have made a first partial implementation of the mine-detection concepts described in the previous paragraph. For these tests we have implemented a threshold on intensity and polarization, and a blob analysis scheme as pattern-recognition classifier.

\section{Threshold on intensity}

The assumption that landmines have intensity values that are different from local surroundings can be exploited using intensity contrast images. As mines can be brighter or darker than the background, the intensity contrast can be a so-called positive or negative contrast with the background. The (normalized) positive intensity contrast image is defined as:

$$
I_{p o s}(x)=\frac{I(x)-\min (I)}{\max (I)-\min (I)}
$$

The (normalized) negative intensity contrast image is defined as:

$$
I_{n e g}(x)=1-\frac{I(x)-\min (I)}{\max (I)-\min (I)}
$$

On both contrast images a threshold can be applied to obtain an image with binary values indicating the detection of landmines. The applied threshold is a parameter of the detection method, and its choice of value depends on the scenario of application and required performance. The positive intensity contrast detections are defined as:

$$
D_{I_{\text {pos }}}(x)=\left\{I_{\text {pos }}(x)\right\}_{t}
$$

The negative intensity contrast detections are defined as: 


$$
D_{I_{n e g}}(x)=\left\{I_{n e g}(x)\right\}_{t}
$$

where the threshold operator is defined as:

$$
\{f(x)\}_{t}=\left\{\begin{array}{l}
1: f(x) \geq t \\
0: f(x)<t
\end{array}\right.
$$

\section{Threshold on polarization}

Landmines that have a smooth artificial surface (and/or have intensity values that are different from local surroundings) can be detected using polarization contrast. Polarization contrast can be defined using the Stokes vector [4], which is a mathematical representation of polarization. Using this representation, optical elements can be described as matrix operators on these vectors. The Stokes vector is constructed by measuring the intensity of four different polarization states:

- $I_{0}$ measures all states equally through an isotropic filter,

- $I_{h}$ measures the intensity through a horizontal linear polarizer,

- $I_{45}$ measures the intensity through a linear polarizer oriented at 45 degrees,

- $I_{L}$ measures the intensity through a polarizer which is opaque for left circular polarization.

Using these measured intensities, the Stokes parameters $I, Q, U, V$ are defined as:

$$
\left\{\begin{array}{l}
I=2 I_{0} \\
Q=2 I_{h}-2 I_{0} \\
U=2 I_{45}-2 I_{0} \\
V=2 I_{L}-2 I_{0}
\end{array}\right.
$$

Often different representations are used for the linear polarization:

$$
\begin{aligned}
L P & =\sqrt{Q^{2}+U^{2}} \\
D o L P & =\frac{L P}{I} \\
\Psi & =\frac{1}{2} \arctan \left(\frac{U}{Q}\right)
\end{aligned}
$$

with $L P$ the amount of linear polarization, DoLP the degree of linear polarization and $\Psi$ the angle of polarization (orientation of the polarization ellipse). The polarization detections are defined as:

$$
D_{p o l}(x)=\{f(I, Q, U)(x)\}_{t}
$$

where $f(I, Q, U)(x)$ is a function that projects the first three elements of the Stokes Vector to a scalar. For example the linear polarization $L P$ (eq. 7), 
with $Q$ and $U$ being the Stokes components that are determined for each pixel. The amount of linear polarization is, in the implementation for the tests, taken as the polarization feature. The normalized polarization contrast image is defined as:

$$
L P_{p o s}(x)=\frac{L P(x)-\min (L P)}{\max (L P)-\min (L P)}
$$

The polarization detections are defined as:

$$
D_{L P_{\text {pos }}}(x)=\left\{L P_{\text {pos }}(x)\right\}_{t}
$$

\section{Combination of intensity and polarization}

For implementation for the tests, we have combined intensity and polarization in the following way:

$$
F(x)=\left\{I_{\text {pos }}(x)\right\}_{t_{1}} \vee\left\{I_{\text {neg }}(x) \cdot L P_{\text {pos }}(x)\right\}_{t_{2}}
$$

The blobs in the resulting binary image $F(x)$ are extracted by means of a connected-component algorithm. Blobs are groups of connected pixels that represent possible landmine detections. Blobs with a size smaller than threshold $t_{3}$ are removed from image $F(x)$. As such, the complete detection procedure has three parameters: thresholds $t_{1}, t_{2}$, and $t_{3}$.

The reason for this combination is as follows: The combination takes a disjunction of the positive intensity and polarization contrast to use either positive intensity or polarization, whichever has more contrast at the appropriate moment. The conjunction between the negative intensity contrast and the polarization is applied for reasons of false-alarm reduction. Furthermore, it is assumed that intensity and polarization are complementary features. This assumption has been validated from the results of the tests.

\section{Detection tests}

To test the detection concepts, detection tests have been performed. This section describes the camera hardware and the detection and performance evaluation software that have been used for the detection tests. These detection tests have been performed using a static setup, looking at a static scene. This section also describes the test procedure, including preparation of the test fields, selection of the test mines and pre-processing of the measured data before detection evaluation. Several factors that are expected to influence the detection performance have been taken into account when setting up and performing the static tests. Factors that are expected to influence the detection results are: 
- Light conditions (elevation and relative azimuth of the sun);

- Distance between camera and mine (number of pixels on mine);

- Mine type;

- Condition of mine surface;

- Burial depth (fraction of mine body visible for camera);

- Vegetation;

- Orientation of top surface of mine (horizontal or tilted).

\subsection{Polarization Measurement Setup}

Generally there are two different approaches used for the measurement of (infrared or visual) polarization. Either time division or spatial division is necessary to measure up to four elements of the Stokes vector using only one focal plane.

With time division, different polarization images are measured sequentially. This is usually performed by mounting a polarization filter in front of the camera and taking a sequence of images with different polarization directions. For measurements of the full (four elements) Stokes vector, a retarder (for instance a quarter wave plate) is rotated followed by a fixed linear polarization filter. This common approach of either rotating a polarizer or a retarder is reported by the majority of literature $[13,14,15]$.

Using spatial division, the different polarization states are measured simultaneously at the cost of reduced spatial resolution. For example, every 4 adjacent pixels of a focal plane array (FPA) are grouped. In front of each of these 4 pixels a different polarization filter is mounted, each with a different orientation $[16,17]$.

When more than one focal plane is available, an optical prism assembly, mounted behind the camera lens can be used to separate an image into three equal components. Each image is captured with a CCD. In front of each CCD element a polarization filter with a different, fixed orientation is mounted. This solution is only known for visible light since visible light CCDs are much cheaper than IR FPAs.

Our approach for the measurement setup is the use of time division and a rotating polarization filter. With this setup only linear polarization can be measured (the first three Stokes components). The measurement set-up consisted of a black and white visible-light camera system, mounted on a tripod. The camera has a frame rate of $25 \mathrm{~Hz}$ and the images are grabbed from the analog video output of the camera using a frame grabber. The rotating polarization filter (Polaroid sheet) was mounted in front of the camera lens. The filter was rotating at a constant speed. A trigger pulse from the rotation set-up started the acquisition of the frame grabber in the computer. Since the camera was running at a fixed frame rate, the time between the frames and thus the rotation of the polarization filter between the different frames is fixed. A recorded sequence (about 56 images) contains at least one full rotation of the filter, which had a rotation time of just over 2 seconds. This

Page: 7 job:version1.2 macro: svmult.cls date/time: 31-Aug-2005/9:50 
camera had a fixed iris and a limited dynamic range, which appeared to be a limitation with shiny mines.

\subsection{Test setup}

\section{Test fields}

In order to perform measurements with different relative sun positions, four test fields were constructed, each in a different direction relative to the camera, which was placed in a central position. Three of the four fields had a stony background; the field in the west direction had a grass background. During data collection, the camera made recordings of the different measurement areas with the camera being rotated on the tripod to view the different areas. From the camera viewpoint, the four areas faced the North, South, East, and West directions.
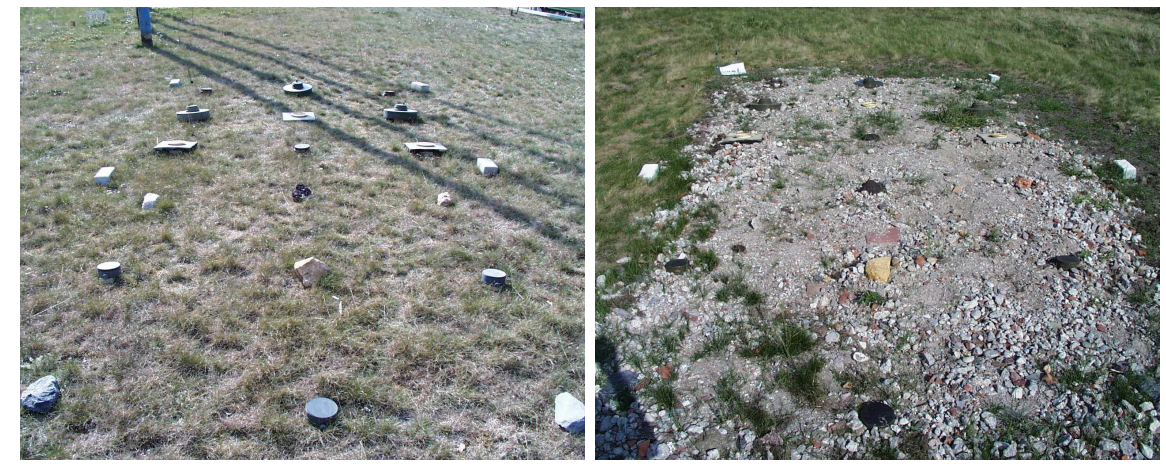

Fig. 1. Two examples of the four measurement areas (facing North, South, East and West). The example on the left is the West measurement area that has grass as background. The other measurement areas have stones as background clutter as shown in the example on the right.

\section{Test mines}

Replicas of four different types of AT mines that appear frequently in mine afflicted countries have been chosen for the tests. One type has a shiny surface (TM62P-3), while the surface of the PMN consists of mat rubber. The surface of the P2 consists of concentric rings which is expected to cause detection difficulties. Quite often only the cap of the mine is visible and not the whole mine body. In the test field only the TM62P-3 mines were surface laid. From the P2 and PRBM-3 only replicas of the detonators were placed (as if the mine body was buried). The PMN AP mines were used since these mines 
have roughly the same diameter as the detonator of an AT mine. These PMNs were surface laid. Each measurement area contains three samples of four mine types and six false alarms. The mines and false alarms were placed in a rectangular area with a width of $3 \mathrm{~m}$ and a length of $6 \mathrm{~m} .18$ objects $(3 \times 6)$ were placed on a square grid. Table 1 shows the layout of the measurement areas.

Table 1. Lay-out of all four measurement areas. The objects are placed on a $1 \mathrm{~m}$ grid. The six false alarms mentioned in this table are boulders with sizes and/or colors that are comparable to the mines that are used.

\begin{tabular}{|c|c|c|c|c|}
\hline & distance to camera & column 1 & column 2 & column 3 \\
\hline row 1 & $13 \mathrm{~m}$ & PRBM-3 & TM62P-3 & PRBM-3 \\
row 2 & $12 \mathrm{~m}$ & TM62P-3 & P2 & TM62P-3 \\
row 3 & $11 \mathrm{~m}$ & P2 & PRBM-3 & P2 \\
row 4 & $10 \mathrm{~m}$ & false alarm & false alarm & false alarm \\
row 5 & $09 \mathrm{~m}$ & PMN & false alarm & PMN \\
row 6 & $08 \mathrm{~m}$ & false alarm & PMN & false alarm \\
\hline
\end{tabular}

Specular reflectivity and thus polarization contrast depends on the smoothness of the mine surface. Measurements were performed with clean mine surfaces and with mine surfaces with some sand on them.

During most measurements, the mines were placed horizontally. However, some measurements were performed with tilted mines, with tilt angles in different directions relative to the camera.

\section{Measurement procedure}

Every 30 minutes during data gathering recordings were taken of each field. The time difference between the recordings of the four fields is less than 1 minute. Most recordings took place between sunrise and sunset. Only a few recordings were taken during the twilight period.

\section{Data pre-processing}

The detection algorithms as described in section 2.2 use Stokes images as input. The recorded data consists of image sequences with different orientations of the rotating polarization filter for the different images in the sequence. Several pre-processing steps are needed to convert the raw data to calibrated Stokes images. 1. Determination of rotation frequency of polarization filter. 2. Correction for intrinsic polarization of camera. 3. Determination of offset angle of filter. 4. Determination of three Stokes parameters for each pixel. 


\subsection{Performance evaluation tools}

The performance of the camera system was evaluated using the ROC curve. In an ROC curve the detection rate is plotted against the false-alarm rate for adjustable optimization parameters. Each working point on the ROC corresponds with a set of values for the thresholds $t_{1}, t_{2}$, and $t_{3}$ (eq. 13). The detection rate is defined as the fraction of detected landmines.

The corresponding number of false alarms per unit area is calculated using the following method: The camera system will be fitted on top of a vehicle that moves forward, and we count multiple false alarms that are on the same horizontal line of the measurement area as one false alarm. This is because the vehicle must stop only once if one or more false alarms are detected on the same horizontal line in front of the vehicle.

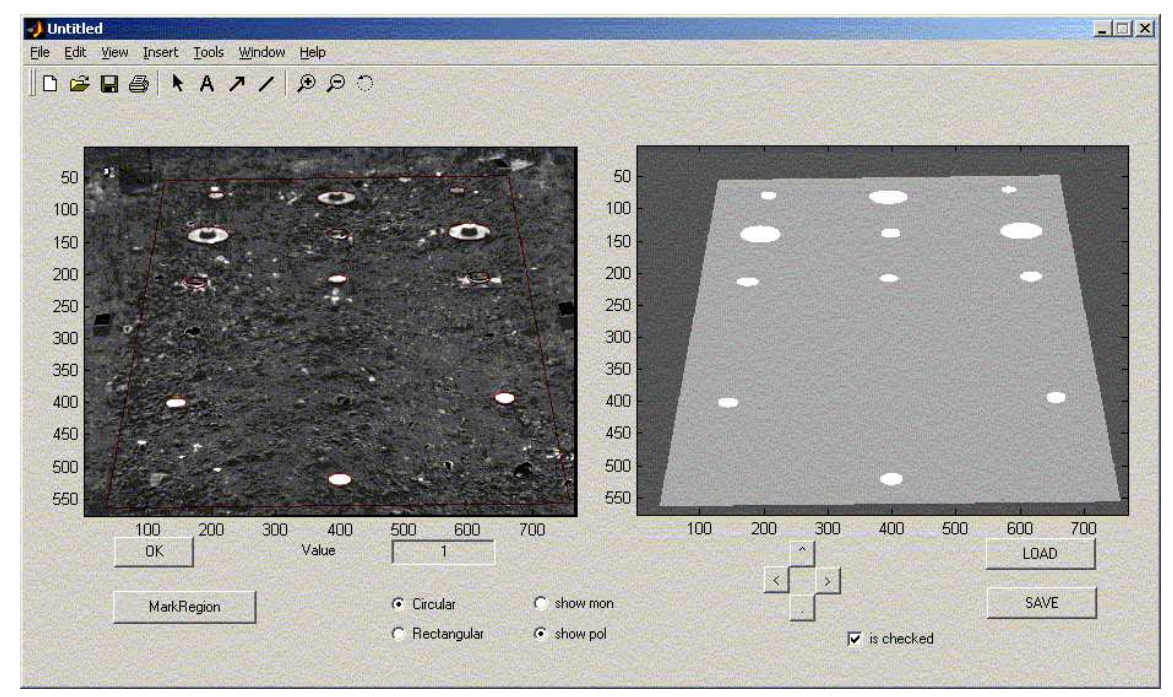

Fig. 2. Performance evaluation tool to make ground truth of measurement areas. The left panel shows the calculated $L P$ image (eq. 7). The light-grey area, which is roughly $3 \mathrm{~m}$ wide and $6 \mathrm{~m}$ deep, in the right panel is used in the detection step. The white circles give the ground truth.

In order to evaluate the performance of the results of the tests a tool was developed to construct the ground-truth of the recorded sequences. Fig. 2 shows the graphical user interface for the tool. In the left window of the tool a visualization of the recorded polarization sequence is shown. Within that window we can mark the region of interest by drawing a boundary box, which appears as a shaded grey area on the right. Detections and/or false alarms outside that box are not taken into account for the results of that sequence. The ground-truth mine positions can be entered by placing a circular or 
rectangular object on the mines on the left. The size of the mine can be altered and the ground-truth mines appear in white on the right.

\section{Test results}

This section presents detection results on recordings made in the late summer period on the test field described in the previous section.

In the following subsections the detection results for different example data sets are presented. The examples are given to show the advantage of using polarization and to assess the influence of several factors (as mentioned in the previous section) to the detection performance as well as to validate the landmine-detection concepts.

\subsection{Polarization versus intensity}

In this subsection we look at the difference in detection results obtained on a data set that was recorded under ideal recording conditions: completely clear sky during the whole day, with a shortwave irradiance that continuously increases from $300 \mathrm{~W} / \mathrm{m}^{2}$ at 9:00 till $750 \mathrm{~W} / \mathrm{m}^{2}$ at 14:00 local time. Fig. 3 shows two ROC curves obtained from the complete data set (all hours, all mines, all viewing directions). In the bottom curve only positive intensity contrast has been used for detection. In the top curve only the amount of linear polarization has been used. From this figure we may conclude that polarization is the most important feature for detection. Although detection based on intensity only has very low performance, the combination of polarization and intensity features generally results in a better ROC. For this specific data set fusion of both features gives an increase in true detections of not more than $5 \%$.

\subsection{Position of the sun}

In this subsection we assess the influence of the relative position of the sun on the performance of the system. Again a data set has been used that was recorded under ideal conditions: completely clear sky during the whole day, with a shortwave irradiance that continuously increases from $250 \mathrm{~W} / \mathrm{m}^{2}$ at 9:00 till $800 \mathrm{~W} / \mathrm{m}^{2}$ at 14:00 local time. For each individual measurement hour and each individual mine type an ROC was calculated. This results in 4 ROC curves per measurement hour. Subsequently in each of those ROC curves the percentage of true detections at a false-alarm rate of $3 \%$ was chosen. Fig. 4(a) shows this percentage of true detections versus the time of measurement for the four mine types. ROC curves were also calculated for each viewing direction (and all mines types combined, with the exclusion of the P2 mine type). Fig. 4(b) shows the percentage of true detections versus the time of

Page: 11 job: version1.2 macro: svmult.cls date/time: 31-Aug-2005/9:50 


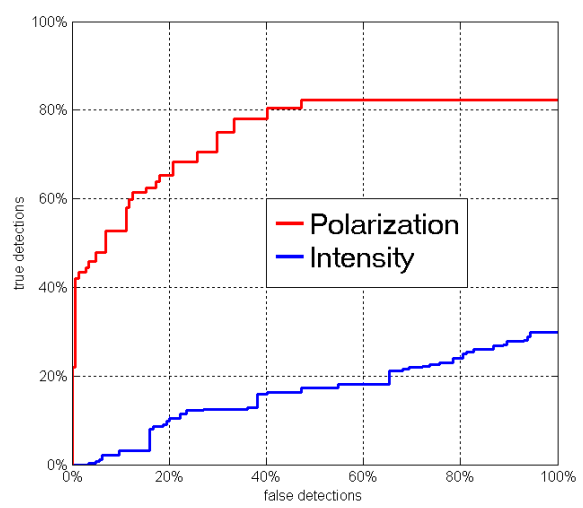

Fig. 3. Two ROC curves obtained from one data set that show a clear difference in detection performance when using polarization features. In the bottom curve only intensity features are used for detection. In the top curve only polarization features are used.

measurement for the four viewing directions. The horizontal dashed lines in the two graphs are the detection results for the whole measurement period (instead of the result of one hour) per mine type (in fig. 4(a)) and per viewing direction (in fig. 4(b)), also with a false-alarm rate of 3\%. From fig. 4(a) we

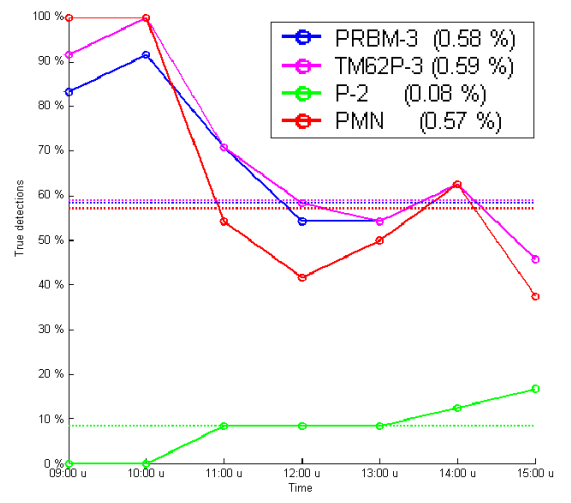

(a)

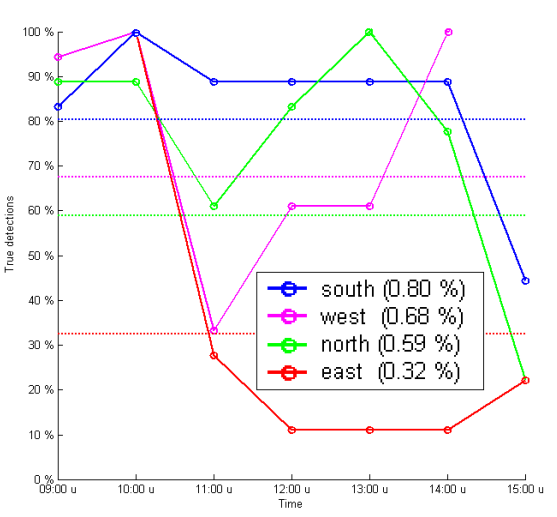

(b)

Fig. 4. (a) Detection rate vs. time of measurement for the four mine types. (b) Detection rate vs. time of measurement for the four viewing directions. In fig. (b) the results for all mine types with the exclusion of the P2 mine type are shown. In both figures a false-alarm rate of $3 \%$ was chosen. The horizontal dashed lines in the figures are the detection results for the whole measurement period (instead of the results of one hour) per mine type (fig. (a)) or per viewing direction (fig. (b)). 
may conclude that detection results per mine type do not differ much, except for the P2 mine, that shows a very low performance. This effect can be explained by the fact that the P2 mine has no flat top surface whereas the other mines have. As a result this specific mine type cannot be detected very well using polarization features.

Furthermore, fig. 4(a) clearly shows the dependency between the recording hour and the detection rate. For all mines (except the P2 mine type) the detection graph shows similar behavior. At 9:00 to 10:00 hours we have top performance, at 12:00 hours the graph shows a local minimum and after 12:00 hours there is increased performance again, with the exception of 15:00 hours. This indicates that there is a relationship between the relative position of the sun and the detection results, given that the recording conditions were ideal.

From fig. 4(b) we may conclude that three out of four viewing directions perform better than $50 \%$ detection with a false-alarm rate of $3 \%$ when we look at the detection results for the whole measurement period (horizontal dashed lines). However, looking at the results per hour, there are some significant variations. This indicates that different parameter settings are used for different hours which do not closely match the global settings for the whole period. From a detailed inspection of the recorded images, we may conclude that a viewing direction directly towards the sun results in the highest polarization contrast of the mines. When the viewing direction is along with the sun, the polarization contrast of the mines is lowest.

\subsection{Influence of clouds}

In this section we look at the detection results for a data set that was recorded under cloudy conditions. The shortwave irradiance changes between $200 \mathrm{~W} / \mathrm{m}^{2}$ and $800 \mathrm{~W} / \mathrm{m}^{2}$ on a time scale of only a few minutes. With the detection results of this data set we assess the influence of cloudy weather on the performance of the system.

Fig. 5(a) presents the detection results for the four mine types. With the exception of the P2 mine the detection results are good. Furthermore, the variation in the results is only significant in the morning and at the beginning of the evening. When we compare the results with the results for the four mine types recorded under a completely clear sky (fig. 4(a)) we can see that there is no drop in performance during the middle of the day. Also the results for the whole period (dashed horizontal lines) are significantly higher.

Fig. 5(b) presents the detection results for the four viewing directions. For all viewing directions the results for the whole period are above the required $50 \%$ detection rate. Note that these results include also the P2 mine which explains the somewhat lower performance in fig. 5(b) when compared to the dashed lines in fig. 5(a) and fig. 4(b). Furthermore, the variation in the results is only significant in the morning and at the beginning of the evening, with the exception of the East direction. When we compare these results with the results for the four viewing directions recorded under a completely clear sky

Page: 13 job: version1.2 macro: svmult.cls date/time: 31-Aug-2005/9:50 


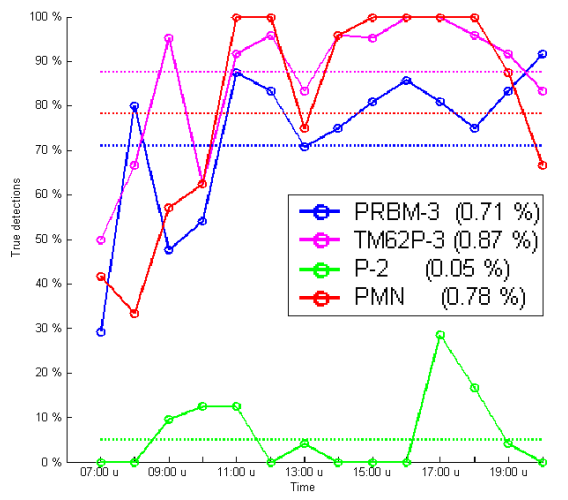

(a)

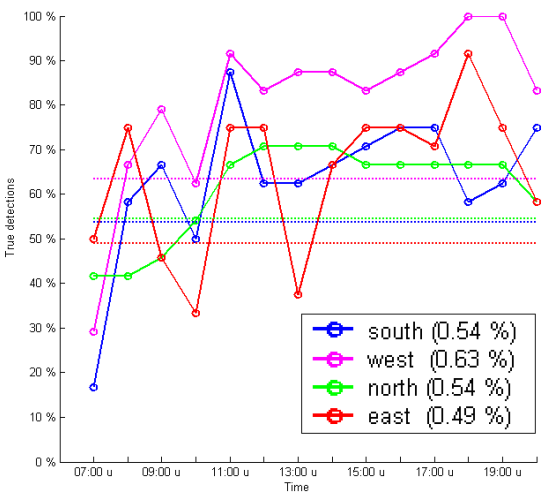

(b)

Fig. 5. (a) Detection rate vs. time of measurement for the four different mine types. (b) Detection rate vs. time of measurement for the four viewing directions. A false-alarm rate of $3 \%$ was chosen.

(fig. 4) we can see that there is far less variation between the four viewing directions. This probably stems from the fact that under cloudy conditions a more uniform light source, that varies less with viewing direction, illuminates the mines. Under the clear sky conditions the sun as a point source gives the highest contribution to the illumination.

\subsection{Surface orientation}

This section describes the results of tests performed using the camera system on tilted mines. The tests were conducted to assess the influence of the mine orientation on polarization results when compared with mines that are not tilted The tests were divided into three parts:

- Mines tilted away from the camera;

- Mines tilted towards the camera;

- Mines tilted to the side.

In all three situations a tilt angle of 10 degrees was used. Only the mines in the $1^{\text {st }}$ and $3^{\text {rd }}$ column of the four measurement areas were tilted (see Table 1). The mines in the inner column remained horizontal to serve as a reference point.

\section{Results for mines tilted away from camera}

Fig. 6 presents the detection results for mines tilted away from the camera (fig. 6(a)) and non-tilted mines (fig. 6(b)) for the four viewing directions. 
Comparison of the dashed lines shows that for each viewing direction the detection results of the mines tilted away from the camera are at least $10 \%$ lower than the detection results of the horizontal mines.

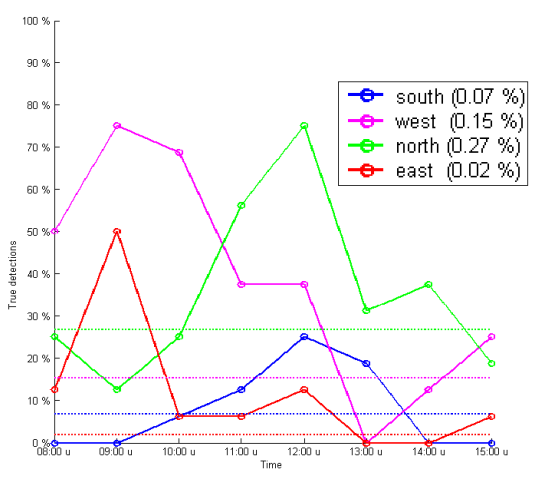

(a)

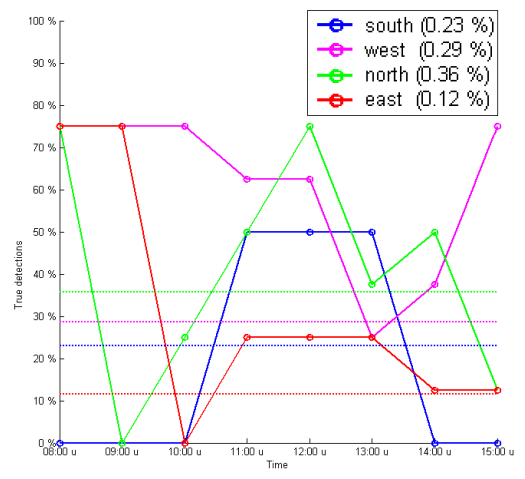

(b)

Fig. 6. Detection rate vs. time of measurement for the four viewing directions. A false-alarm rate of $3 \%$ was chosen. (a) Results for mines tilted away from the camera. (b) Results for the non-tilted mines.

\section{Results for mines tilted towards the camera}

Fig. 7 presents the detection results for mines tilted towards the camera (fig. $7(\mathrm{a})$ ) and non-tilted mines (fig. $7(\mathrm{~b})$ ) for the four viewing directions. Comparison of the dashed lines shows that detection results for mines tilted towards the camera only deteriorated for the North viewing direction when compared with the horizontal mines of the same data set. The other viewing directions showed improved performance. The East viewing direction performs poorly for tilted and non-tilted mines because of shadows in the morning imagery and background clutter in the recordings later on the day.

\section{Results for mines tilted along the viewing direction}

Fig. 8 presents the detection results for mines tilted along the viewing direction (fig. 8(a)) and non-tilted mines (fig. 8(b)) for the four viewing directions. Comparison of the dashed lines shows that except for the north direction detection results deteriorate for tilted mines when compared with the non-tilted mines. However the results per hour of the west direction do not differ much. 


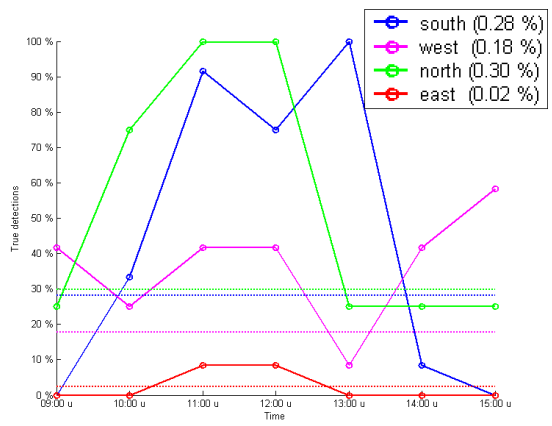

(a)

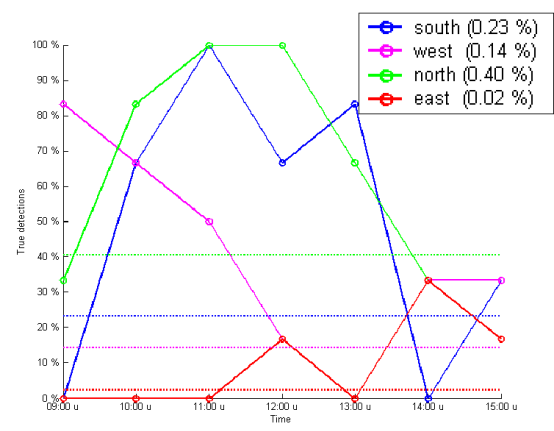

(b)

Fig. 7. Detection rate vs. time of measurement for the four viewing directions. A false-alarm rate of $3 \%$ was chosen. (a) Results for mines tilted towards the camera. (b) Results for the non-tilted mines.

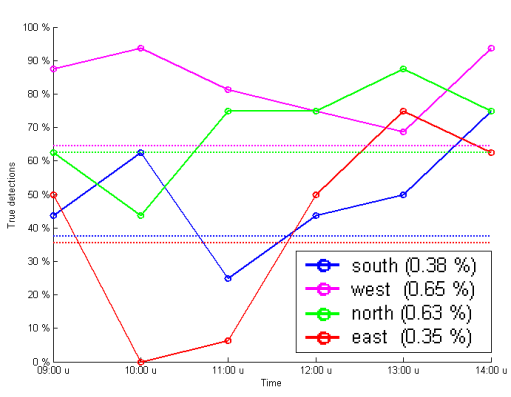

(a)

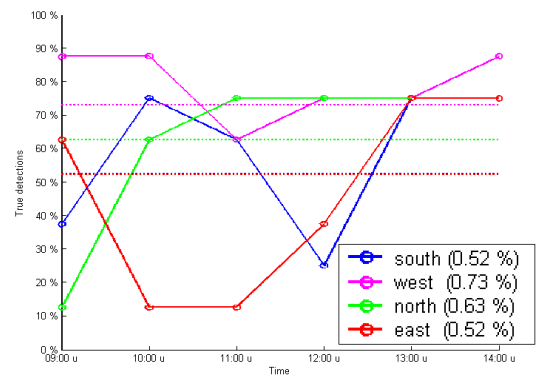

(b)

Fig. 8. Detection rate vs. time of measurement for the four viewing directions. A false-alarm rate of $3 \%$ was chosen. (a) Results for mines tilted along the viewing direction. (b) Results for the non-tilted mines.

\section{Conclusions}

In this chapter we have described the usage of polarization features of visible light for automatic landmine detection.

Given the results of the conducted experiments the following conclusions can be drawn:

- Combination of intensity and polarization gives the best performance of an automatic landmine detection system.

- Performance depends on viewing direction, relative to the position of the sun. Looking towards the sun (i.e., when the sun is in a sector of \pm 45 degrees when looking in the forward motion direction) seems to be the best viewing direction. This is illustrated by the results for some of the data 
sets from the West viewing direction (see for example the magenta curve in fig. 4(b)). Note that for the West viewing direction the background clutter is far less (grass field) when compared with the other directions, which have debris as background clutter.

- The East viewing direction performs less well because of the position of the sun relative to the viewing direction during most measuring hours.

- Performance depends on the time of day and shows large variations, which may be partly due to weather conditions, shadows and a fixed camera iris. A camera with a larger dynamic range could solve part of these variations.

- Performance depends on the type of mine: detection results do not differ much for the mines used in the test, with the exception of the P2 mine that shows a very low performance. This can be explained by the fact that the P2 mine has no flat top surface, and as such, cannot be detected very well using polarization.

- Detection of tilted mines proves to be more difficult, except for mines tilted towards the camera.

\section{References}

1. B. Gros and C. Bruschini, "Sensor technologies for the detection of antipersonnel mines: a survey of current research and system developments," in Proceedings of the International Symposium on Measurement and Control in Robotics (ISMCR'96), pp. 509-518, (Brussels, Belgium), May 1996.

2. F. Cremer, K. Schutte, J. G. M. Schavemaker, and E. den Breejen, "A comparision of decision-level sensor-fusion methods for anti-personnel landmine detection," Information Fusion 2, pp. 187-208, Sept. 2001.

3. J. G. Schavemaker, E. den Breejen, K. W. Benoist, K. Schutte, P. Tettelaar, M. de Bijl, P. J. Fritz, L. H. Cohen, W. van der Mark, and R. J. Chignell, "LOTUS field demonstration of integrated multisensor mine detection system in Bosnia," in Proc. SPIE Vol. 5089, Detection and Remediation Technologies for Mines and Minelike Targets VIII, R. S. Harmon, J. T. Broach, and J. John H. Holloway, eds., Proceedings of SPIE 5089, pp. 1324-1335, SPIE - The International Society for Optical Engineering, (Bellingham (WA), USA), Sept. 2003.

4. E. Hecht, Optics, Addison-Wesley Publishing Company, Inc., Reading (MA), USA, second ed., 1987.

5. F. Cremer, W. de Jong, and K. Schutte, "Infrared polarisation measurements and modelling applied to surface laid anti-personnel landmines," Optical Engineering 41, pp. 1021-1032, May 2002.

6. F. Cremer, W. de Jong, K. Schutte, A. Yarovoy, V. Kovalenko, and R. Bloemenkamp, "Feature level fusion of polarimetric infrared and gpr data for landmine detection," in International Conference on Requirements and Technologies for the Detection, Removal and Neutralization of Landmines and UXO, H. Sahli, A. Bottoms, and J. Cornelis, eds., pp. 638-642, (Brussels, Belgium), Sept. 2003. 
7. F. Cremer, Polarimetric infrared and sensor fusion for the detection of landmines. Ph.D. dissertation, Technische Universiteit Delft, Delft, Nov. 2003.

8. Technical Note 09.50/01; Guide to mechanical mine clearance/ground preparation using commercial tractors and front loaders. http://www.mineactionstandards.org/tnma_list.htm, 2002. Version 1.0.

9. J. G. M. Schavemaker, F. Cremer, K. Schutte, and E. den Breejen, "Infrared processing and sensor fusion for anti-personnel land-mine detection," in Proceedings of IEEE Student Branch Eindhoven: Symposium Imaging, pp. 61-71, (Eindhoven, the Netherlands), May 2000.

10. M. Swain and D. Ballard, "Color indexing," International Journal of Computer Vision 7, pp. 11-32, Jan. 1991.

11. W. A. C. M. Messelink, K. Schutte, A. M. Vossepoel, F. Cremer, J. G. M. Schavemaker, and E. den Breejen, "Feature-based detection of landmines in infrared images," in Proc. SPIE Vol. 4742, Detection and Remediation Technologies for Mines and Minelike Targets VII, J. T. Broach, R. S. Harmon, and G. J. Dobeck, eds., Proceedings of SPIE 4742, pp. 108-119, SPIE - The International Society for Optical Engineering, (Bellingham (WA), USA), Apr. 2002.

12. C. F. Olson, "Adaptive-scale filtering and feature detection using range data," IEEE Transactions on Pattern Analysis and Machine Intelligence 22, Sept. 2000 .

13. T. Rogne, S. Stewart, and M. Metzler, "Infrared polarimetry: what, why, how and the way ahead," in Proc. of Third NATO IRIS Joint Symposium, pp. 357368, (Quebec, Canada), Oct. 1998.

14. K. P. Bishop, H. D. McIntire, M. P. Fetrow, and L. McMackin, "Multi-spectral polarimeter imaging in the visible to near IR," in Proc. SPIE Vol. 3699, Targets and Backgrounds: Characterization and Representation V, W. R. Watkins, D. Clement, and W. R. Reynolds, eds., pp. 49-57, (Orlando (FL), USA), Apr. 1999.

15. M. H. Smith, "Optimizing a dual-rotating-retarder Mueller matrix polarimeter," in Proc. SPIE Vol. 4481, Polarization and Remote Sensing IV, W. G. Egan and M. J. Duggin, eds., Proceedings of SPIE 4481, pp. 31-36, SPIE The International Society for Optical Engineering, (San Diego (CA), USA), July 2001.

16. F. A. Sadjadi and C. S. L. Chun, "Application of a passive polarimetric infrared sensor in improved detection and classification of targets," International Journal of Infrared and Millimeter Waves 19(11), pp. 1541-1559, 1998.

17. G. P. Nordin, J. T. Meier, P. C. Deguzman, and M. W. Jones, "Micropolarizer array for infrared imaging polarimetry," J. Opt. Soc. Am. A 16, pp. 1168-1174, May 1999. 